\title{
Extracto etanólico de Baccharis genistelloides (carqueja) sobre el cáncer de colon inducido con 1,2-dimetilhidrazina en ratas ${ }^{*}$
}

\section{Effect of Baccharis genistelloides (carqueja) ethanol extract on 1,2-dimethylhydrazine-induced rodent's colon cancer}

\author{
Hugo Justil ${ }^{1}$, Jorge Arroyo ${ }^{2,3}$, José Valencia ${ }^{1}$ \\ ${ }^{1}$ Facultad de Farmacia y Bioquímica, Universidad Nacional Mayor de San Marcos \\ ${ }^{2}$ Instituto de Investigaciones Clínicas, Facultad de Medicina, Universidad Nacional Mayor de San Marcos. Lima, Perú. \\ ${ }^{3}$ Laboratorio de Farmacología, Facultad de Medicina, Universidad Nacional Mayor de San Marcos. Lima, Perú. \\ * Trabajo de tesis para optar al grado académico de Magister en Farmacología Experimental. Facultad de Medicina, Universidad Nacional Mayor de San Marcos.
}

\section{Resumen}

Introducción: Se tiene conocimiento que el extracto etanólico de las hojas de Baccharis genistelloides puede reducir la aparición del cáncer gástrico y es marcador de estrés oxidativo. Objetivos: Determinar la eficacia quimioprotectora del extracto etanólico de las hojas de Baccharis genistelloides (EEBG) en el cáncer de colon inducido por 1,2 - dimetilhidracina (DMH) en ratas machos. Diseño: Experimental. Institución: Laboratorio de Farmacología, Facultad de Medicina, UNMSM, Lima, Perú. Material biológico: Carqueja recolectada en Huancayo, Junín, y ratas machos de $145+15 \mathrm{~g}$. Intervenciones: Se indujo tumores intestinales con inyección subcutánea semanal de DMH durante 22 semanas, a $20 \mathrm{mg} / \mathrm{kg}$. Se formó seis grupos: Grupo 1 suero fisiológico; Grupo 2100 mg/kg EEBG; Grupo 3 DMH; Grupo 4 DMH más 100 mg/kg de EEBG; Grupo 5 DMH más $250 \mathrm{mg} / \mathrm{kg}$ de EEBG; y, Grupo 6 DMH más 500 mg/kg de EEBG. Finalmente, se extrajo muestra de sangre para determinar el nivel de malondialdehido y óxido nítrico. Principales medidas de resultados: Quimioprotección. Resultados: El estudio histopatológico mostró quimioprotección de los grupos que recibieron tratamiento con EEBG frente al grupo que no recibió tratamiento, presentando mejor quimioprotección a dosis de $500 \mathrm{mg} / \mathrm{kg}$, donde el cáncer fue pobremente diferenciado, presentando adenomas, frente a adenocarcinoma in situ y adenocarcinoma a dosis de $250 \mathrm{mg} / \mathrm{kg}$ y 100 $\mathrm{mg} / \mathrm{kg}$; el potencial de oxidación de lipoproteínas fue reducido en los grupos que recibieron tratamiento con EEBG frente a los no tratados, mostrando mayor efecto la dosis de $500 \mathrm{mg} / \mathrm{kg}$; los niveles de óxido nítrico también mostraron una mayor disminución a la dosis de $500 \mathrm{mg} / \mathrm{kg}$. Conclusiones: En ratas, el extracto etanólico de Baccharis genistelloides tiene efecto quimioprotector sobre el cáncer de colon inducido con 1,2-dimetilhidracina.

Palabras clave: Baccharis; cáncer; colon; 1,2-dimetilhidrazina.

\begin{abstract}
Introduction: Baccharis genistelloides leaves ethanol extract may reduce gastric cancer development and is an oxidative stress marker. Objectives: To determine the chemoprotector efficacy of Baccharis genistelloides (EEBG) leaves ethanol extract in 1,2-dimethylhydrazine (DMH)-induced colon cancer in male rats. Design: Experimental. Setting: Pharmacology Laboratory, Faculty of Medicine, Universidad Nacional Mayor de San Marcos, Lima, Peru. Biological material: Carqueja collected in Huancayo, Junin, and $145+15 \mathrm{~g}$ male rats. Interventions: Intestinal tumors were induced by weekly subcutaneous injection of DMH for 22 weeks at $20 \mathrm{mg} / \mathrm{kg}$. Six groups were formed: Group 1 saline, Group $2100 \mathrm{mg} /$ kg EEBG; Group 3 DMH; Group 4 DMH plus 100 mg/kg EEBG; Group 5 DMH plus 250 mg/kg EEBG; Group 6 DMH plus 500 mg/kg EEBG. Finally, blood samples were drawn to determine malondialdehyde and nitric oxide level. Main outcome measures: Chemoprotection. Results: Histopathology showed chemoprotection in groups treated with EEBG versus the group without treatment, showing better chemoprotection at $500 \mathrm{mg} / \mathrm{kg}$ doses where cancer was poorly differentiated and presenting adenomas, compared with adenocarcinoma in situ and adenocarcinoma respectively at $250 \mathrm{mg} / \mathrm{kg}$ and $100 \mathrm{mg} / \mathrm{kg}$ doses. Lipoproteins oxidation potential was reduced in groups treated with EEBG compared to those untreated especially with the $500 \mathrm{mg} / \mathrm{kg}$ dose; nitric oxide levels also decreased more with $500 \mathrm{mg} / \mathrm{kg}$ dose. Conclusions: Baccharis genistelloides ethanol extract has chemoprotective effects on 1,2-dimethylhydrazine-induced rodent's colon cancer.
\end{abstract}

Key words: Baccharis; neoplasms; colon; 1,2-dimethylhydrazine.

\section{INTRODUCCIÓN}

El cáncer de colon muestra una marcada incidencia y mortalidad en ambos sexos (1), siendo un delicado problema de salud humana, hecho que es favorecido por la resistencia farmacológica y los marcados efectos adversos de los agentes usados clínicamente. Por ello es la búsqueda constante de productos naturales con eficacia demostrada y con mínimos efectos adversos.

Si bien es cierto que al presente no se ha obtenido información de Baccharis genistelloides (carqueja) sobre estudios relacionados con el cáncer de colon, pero sí se tiene sobre cáncer gástrico inducido en ratas, resultando en una reducción del cáncer gástrico y de marcador de estrés oxidativo con extracto etanólico de las hojas de Baccharis genistelloides ${ }^{(2)}$. Por ello, esta investigación es propuesta en virtud a la presencia de flavonoides y compuestos fenólicos en la planta ${ }^{(3)}$.

Una amplia variedad de flavonoides y compuestos fenólicos han mostrado poseer efectos anticarcinogénicos y antimutagénicos. Es así que flavonoides, como la quercetina presente en el género Baccharis, han mostrado tener efecto antioxidante y anticancerígeno ${ }^{(4)}$.

La mutación del gen $\mathrm{K}$-ras es el inicio en el proceso de formación de células cancerígenas y por ende la proliferación de estas; además, esta mutación ocurre antes de la formación de adenocarcinomas ${ }^{(5)}$. Por su lado, el óxido nítrico (NO) refuerza la activación de la mutación del gen $\mathrm{K}$-ras ${ }^{(6)} \mathrm{y}$ a su vez favorece la angiogénesis y lipoperoxidación en los tumores colónicos inducidos en $\operatorname{ratas}^{(7)}$. 
Los objetivos del presente trabajo fueron: 1) Determinar la eficacia quimioprotectora del extracto etanólico de las hojas de Baccharis genistellodes sobre el cáncer de colon inducido en ratas; 2) Determinar la dosis efectiva media del extracto etanólico de las hojas de Baccharis genistelloides que puede inhibir el desarrollo de cáncer de colon inducido en ratas; 3) Determinar los niveles de óxido nítrico al administrar extracto etanólico de las hojas de Baccharis genistelloides en ratas con cáncer de colon inducido; 4) Determinar el nivel de un marcador de estrés oxidativo al administrar extracto etanólico de las hojas de Baccharis genistelloides en ratas con cáncer de colon inducido; y, 5) Observar posibles cambios histopatológicos por administración diaria de extracto etanólico de las hojas de Baccharis genistelloides en ratas normales, durante un periodo de 23 semanas.

\section{MÉTODOS}

El material biológico consistió en ratas albinas de cepa Holtzman, con peso al inicio de la investigación de $145+/-15 \mathrm{~g}$, de 3 meses de edad, machos, procedentes del Bioterio del Instituto Nacional de Salud del Ministerio de Salud, mantenidos en un ambiente a temperatura de $21^{\circ} \mathrm{C}$, con dieta y agua a libertad. La planta medicinal consistió en las hojas de Baccharis genistelloides (carqueja).

La planta entera fue recolectada en el Anexo de Ocopilla, distrito de Huancayo, Provincia de Huancayo, Departamento de Junín, en el mes de febrero, y posteriormente fue identificada en el Museo de Historia Natural de la Universidad Nacional Mayor de San Marcos. La planta entera fue secada bajo sombra y en lugar fresco.

En la preparación del extracto etanólico, según Lock, $1994{ }^{(8)}$, la planta entera fue desecada bajo sombra durante un periodo de 2 semanas; luego se separó las raíces de la planta. Las hojas y tallos fueron molidos en un molino a cuchillas eléctrico, hasta obtener polvo fino y homogéneo. La preparación de extractos fue por maceración etanólica, donde 5 $\mathrm{kg}$ de polvo de planta total seca fueron macerados en etanol al 96\%, durante
8 días, con agitaciones diarias de 10 minutos, en envases de color ámbar y protegidos de la luz solar directa. Se filtró con papel filtro de porosidad mediana y se concentró a sequedad, en estufa a $40^{\circ}$, obteniéndose un residuo seco de peso constante, al que se le denominó extracto etanólico seco.

Con relación a la marcha fitoquímica, según Lock, $1994^{(8)}$, a cada reacción de identificación de metabolitos secundarios presentes en el extracto etanólico seco se realizó con los reactivos específicos (5 mg del extracto problema con 5 gotas de reactivo), reportando en los resultados la presencia o ausencia del metabolito.

La inducción de cáncer de colon en ratas Holtzmann por 1,2-dimetilhidrazina (DMH) se realizó según Namasivayam y col., $2004^{(9)}$, con modificaciones en pesos de ratas y el tiempo de administración de DMH. Se empleó 48 ratas Holtzman de 3 meses de edad, con pesos $130 \mathrm{~g}$ a $160 \mathrm{~g}$, aproximadamente, agrupadas en seis grupos de ocho cada uno. Los pesos de los animales fueron evaluados semanalmente durante las 23 semanas que duró el estudio. Los tumores intestinales fueron inducidos mediante una inyección subcutánea semanal de 20 miligramos de 1,2-dimetilhidrazina $(\mathrm{DMH})$ por kilogramo de peso corporal, durante 17 semanas. La DMH fue preparada como una solución que contenía 400 mg de DMH disuelta en $100 \mathrm{~mL}$ de agua destilada estéril, conteniendo 37 mg de EDTA como agente estabilizador, y mantenida dicha solución a pH 6,5 con hidróxido de sodio.

La administración del extracto etanólico de las hojas de Baccharis genistelloides (carqueja) (EEBG) y suero fisiológico fue por vía oral, haciendo uso de una sonda metálica, iniciándose una semana antes de la primera inoculación del DMH hasta las 23 semanas que duró el estudio.

Para la determinación de la eficacia quimioprotectora del extracto etanólico de las hojas de Baccharis genistelloides, se asignó los tratamientos para cada grupo de la siguiente manera: el Grupo 1 (control) recibió suero fisiológico, el Grupo 2 recibió $100 \mathrm{mg} / \mathrm{kg}$ EEBG, el Grupo 3 recibió DMH, el Grupo 4 DMH más 100 mg/kg de EEBG, el Grupo 5 DMH más $250 \mathrm{mg} / \mathrm{kg}$ de EEBG y el Grupo 6 DMH más $500 \mathrm{mg} / \mathrm{kg}$ de EEBG.

En la semana 22, los animales fueron sacrificados por dislocación cervical, extrayéndose el intestino, hígado y riñón, los cuales fueron conservados en una solución de formol al 10\%; luego, se realizó los cortes histológicos de cada órgano, para su respectivo estudio histopatológico.

El colon fue retirado y lavado suavemente con solución salina para remover la sangre y los detritos adheridos al tejido; zonas de masa tumoral fueron fijadas en solución de formol al 10\%, tamponado por 7 días; luego, se seleccionó partes de $3-5 \mu \mathrm{m}$ y fueron incluidas en parafina, $\mathrm{y}$ posteriormente teñidas con hematoxilina y eosina. Los criterios de evaluación fueron en base a las observaciones microscópicas: adenoma, adenocarcinoma Insitu, adenocarcinoma glandular, adenocarcinoma glandular y escamoso.

Para determinar la dosis efectiva media (DEM), se utilizó el método de regresión Probits, usando programa SPSS y teniendo en cuenta los grupos 3, 4, 5 y 6 , a quienes se les administró DMH y dosis crecientes de extracto etanólico de las hojas de Baccharis genistelloides + DMH (100 mg/kg, 250 mg $/ \mathrm{kg}$ y $500 \mathrm{mg} / \mathrm{kg}$ ). La variable evaluada fue el número de ratas protegidas (sin presencia de lesión) por cada grupo.

Para la determinación de los niveles de óxido nítrico, según Katrina y col., $2001^{(10)}$, se extrajo muestra de sangre por punción cardiaca de cada una de las ratas tratadas, $5 \mathrm{~mL}$ por muestra, las cuales fueron recolectadas en tubos heparinizados, homogenizadas mediante agitación constante y depositadas en un recipiente con agua helada para estabilizarlas, durante 10 minutos. Se procedió a centrifugar durante 15 minutos, a $3500 \mathrm{rpm}$, para separar los elementos formes del plasma. Se extrajo el plasma mediante micropipetas y se depositó en tubos numerados correlativamente.

Las muestras de plasma obtenidas fueron sometidas a desproteinización en medio alcalino, mediante la adición de las sustancias en la siguiente proporción: 
Plasma

$0,8 \mathrm{~mL}$

$\mathrm{H}_{2} \mathrm{O}$

$0,6 \mathrm{~mL}$

$\mathrm{Na}(\mathrm{OH}) 1 \mathrm{M}$

$0,1 \mathrm{~mL}$

$\mathrm{ZnSO}_{4} 30 \%$

$0,1 \mathrm{~mL}$

Entre la adición de la sustancia alcalina, $\mathrm{Na}(\mathrm{OH})$, y la sustancia atrapadora de proteínas, $\mathrm{ZnSO}_{4}$, hubo un intervalo de 5 minutos. Posteriormente, se ejerció agitación constante por 10 minutos, obteniéndose una suspensión lechosa. Se procedió a centrifugar durante $15 \mathrm{mi}$ nutos, a 3500 rpm, y se separó el plasma desproteinizado (incluido los controles) en una nueva batería.

Para el análisis espectrofotométrico, a la batería conteniendo plasma desproteinizado se le adicionó $\mathrm{Zn}$ metálico, dejándolo reposar durante 1 hora y 45 minutos, de manera de reducir los nitratos en nitritos. Transcurrido el tiempo, se centrifugó durante 15 minutos a 3500 rpm, para separar el precipitado de la nueva solución de plasma. A la nueva solución se le adicionó reactivo de Griess A (ácido sulfanílico al 1\% en ácido fosfórico al 5\%); 5 minutos después, reactivo de Griess B (N-1-naftiletilendiamina al $0,1 \%$ en agua destilada). Se dejó reposar por 10 minutos, tornándose de un grisáceo más claro. Finalmente, se procedió a la lectura en el espectrofotómetro UV. VIS (LaboMed) a una longitud de onda de $546 \mathrm{~nm}$. Las lecturas de absorbancia obtenidas fueron transformadas a concentración plasmática en $\mu \mathrm{M}$.

Para la determinación de un marcador de estrés oxidativo, de la muestra de sangre extraída por punción cardiaca de cada una de las ratas tratadas, se separó el suero, y el estrés oxidativo se midió mediante la prueba de lipoperoxidación, siguiendo la técnica de Buege y col., 1978 (11), midiendo la producción de malondialdehído (MDA) expresado en nmol/ $\mathrm{mL}$ en plasma que, al reaccionar con el ácido tiobarbitúrico (TBA), forma un complejo coloreado que será leído a 535 $\mathrm{nm}$ en un espectrofotómetro UV-VIS (LaboMed).

Los análisis de los resultados de óxido nítrico, estrés oxidativo, evolución de pesos fueron presentados en un cuadro descriptivo, considerando media, des-
Tabla 1. Marcha fotoquímica del extracto etanólico de las hojas de Baccharis genistelloides.

\begin{tabular}{|c|c|c|c|c|c|c|c|c|}
\hline & Alcaloides & $\begin{array}{l}\text { Flavi- } \\
\text { noides }\end{array}$ & $\begin{array}{l}\text { Sapo- } \\
\text { ninas }\end{array}$ & $\begin{array}{l}\text { Compuestos } \\
\text { fenólicos }\end{array}$ & Taninos & $\begin{array}{l}\text { Esteroides } \\
\text { terpénicos }\end{array}$ & $\begin{array}{l}\text { Aminoácidos } \\
\text { libres }\end{array}$ & $\begin{array}{l}\text { Glicó- } \\
\text { sidos }\end{array}$ \\
\hline Dragendorf & 3 & & & & & & & \\
\hline Shinoda & & 2 & & & & & & \\
\hline Espuma & & & 1 & & & & & \\
\hline Tricloruro férrico & & & & 2 & & & & \\
\hline Gelatina & & & & & 2 & & & \\
\hline Lieberman-Burchard & & & & & & 0 & & \\
\hline Ninhidrina & & & & & & & 0 & \\
\hline Molish (alfa naftol) & & & & & & & & 1 \\
\hline
\end{tabular}

Donde: 0) ausencia, 1) poca cantidad 2) regular cantidad 3) abundante cantidad. viación estándar, intervalo de confianza, mínimos y máximos. También, se aplicó análisis de homogeneidad de varianza, así como análisis de varianza (Anova), realizando comparaciones múltiples si en los resultados existía diferencia significativa $(p<0,05)$ y análisis de varianza con múltiples comparaciones de Turkey en caso de que la varianza fuera heterogénea.

\section{RESULTADOS}

Taxonómicamente, la planta medicinal fue identificada como Baccharis genistelloides (Lamarck) Persoon. El rendimiento fue de 23,5 gramos, de 250 gramos de plantas enteras secas y molidas, equivalente al
9,4\% de las plantas enteras. Al realizar el análisis fotoquímico, se identificó a los flavonoides, compuestos fenólicos y taninos en regular cantidad, mientras que los alcaloides estaban en abundante cantidad (tabla 1).

La figura 1 representa la evolución del crecimiento con respecto al peso corporal durante todo el estudio. En la semana veintidós, se observó que los pesos ganados por el grupo normal era mayor que el grupo EEBG $100 \mathrm{mg} / \mathrm{kg}$, a su vez mayor al grupo DMH + EEBG 500 $\mathrm{mg} / \mathrm{kg}$, que fue mayor al grupo DMH + EEBG $250 \mathrm{mg} / \mathrm{kg}$, mayor al grupo DMH + EEBG $100 \mathrm{mg} / \mathrm{kg}$ y mayor al grupo $\mathrm{DMH}$.

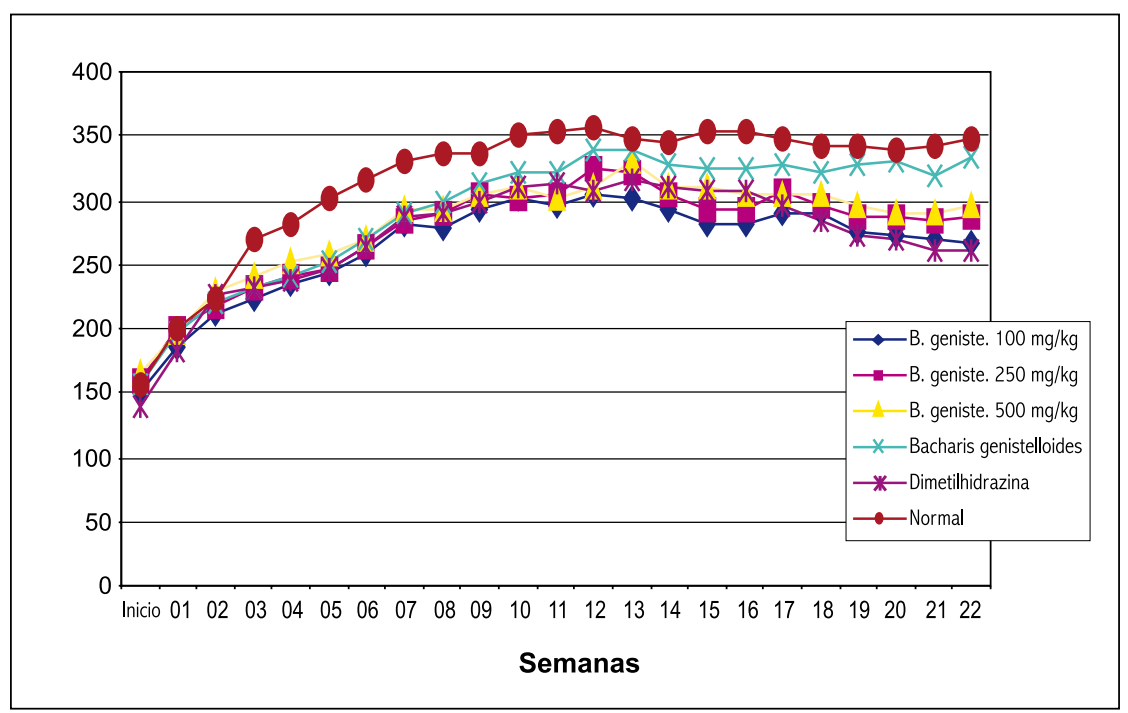

Figura 1. Evolución de crecimiento durante las veintidós semanas, tomando como referencia el peso corporal cada semana. 


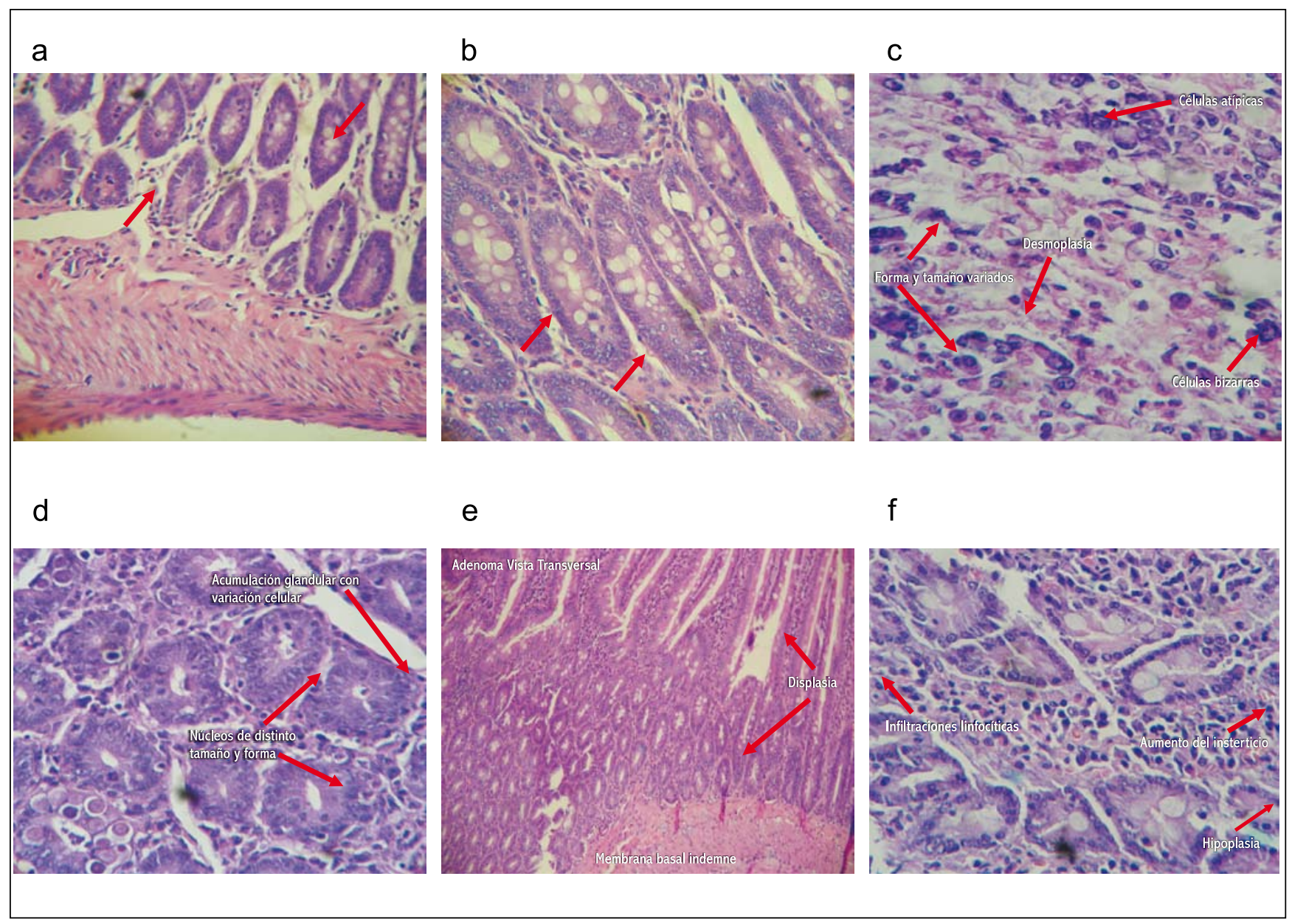

Figura 2. a(40x), b(40x), c(10x), d(40x), e(10x), f(10x). Ver explicación en el texto.

En la figura 2 se observa los resultados histopatológicos; en la figura 2a se muestra al grupo control, observándose un colon sin alteraciones significativas, estructuras celulares conservadas; en la figura $2 \mathrm{~b}$ se muestra al grupo que recibió $100 \mathrm{mg} / \mathrm{kg}$ de EEBG, donde se observa el colon sin alteraciones significativas, células hipertróficas sin anormalidades; en la figura 2c está el grupo que recibió solo DMH, donde el colon presenta adenocarcinoma indiferenciado (glandular y escamoso); en la figura $2 \mathrm{~d}$ se muestra al grupo que recibió $\mathrm{DMH}+100 \mathrm{mg} / \mathrm{kg}$ de EEBG, donde se observa adenocarcinoma glandular en el colon; en la figura 2e está el grupo que recibió $\mathrm{DMH}+250 \mathrm{mg} / \mathrm{kg}$ de EEBG, en el que el colon presenta adenoma, doble fila de célula (adenocarcinoma in situ, porque no ha pasado la membrana basal), desmoplasia; y, finalmente, en la figura $2 \mathrm{f}$ se muestra al grupo que recibió
$\mathrm{DMH}+500 \mathrm{mg} / \mathrm{kg}$, donde se observa al colon con adenoma, zonas de hipoplasia glandular, abundantes macrófagos, infiltración del parénquima por células mononucleares (reacción crónica al tóxico).

Los valores de dosis efectiva media (DEM) son descritos en la tabla 2 y figura 3 .

El EEBG disminuyó los niveles plasmáticos de nitritos en las ratas tratadas con DMH 20 mg/kg (tabla 3).

Se observó que la dosis de $500 \mathrm{mg} / \mathrm{kg}$ de EEBG disminuyó mejor los niveles nitritos. Estos niveles de nitritos en los animales tratados solamente con DMH fueron superiores a todos los grupos tratados con EEBG (figura 4).

La tabla 4 muestra la concentración plasmática de malondialdehído (MDA) en el grupo normal y grupos experimentales. Se observa además que el grupo que recibió las dosis de $500 \mathrm{mg} / \mathrm{kg}$ de EEBG disminuyó mejor la concentración plasmática de MDA (figura 5).

\section{DISCUSIÓN}

Los estudios sobre la actividad antitumoral de Baccharis genistelloides, específicamente sobre el cáncer de colon, son escasos.

Hasta el momento se ha comunicado que el extracto etanólico de Baccharis genistelloides (EEBG) ha mostrado tener un efecto quimioprotector in vivo de neoplasia gástrica inducida en ratas machos (2); así mismo, se encuentra estudios in vitro de especies del género Baccharis, en los que se inhibió la lipoperoxidación, disminuyendo los niveles de radicales 
Tabla 2. Determinación de la dosis efectiva media del extracto etanólico de las hojas de Baccharis genistelloides (carqueja) en ratas con cáncer de colon inducidas con DMH, tomando como referencia el número de ratas lesionadas y de ratas protegidas.

\begin{tabular}{|c|c|c|c|c|c|c|}
\hline Tratamiento & $n$ & $\begin{array}{c}\mathrm{N}^{0} \\
\text { lesionadas }\end{array}$ & $\begin{array}{c}\mathrm{N}^{0} \\
\text { protegidas }\end{array}$ & Resultados & $\begin{array}{c}\% \\
\text { Lesión }\end{array}$ & $\begin{array}{c}\% \\
\text { Protección }\end{array}$ \\
\hline DMH + suero & 8 & 8 & 0 & $\begin{array}{l}\text { Adenocarcinoma glandular } \\
\text { y escamoso }\end{array}$ & 100 & 0 \\
\hline $\mathrm{DMH}+\mathrm{EEBG} 100 \mathrm{mg} / \mathrm{kg}$ & 8 & 6 & 2 & Adenocarcinoma glandular & 75 & 25 \\
\hline $\mathrm{DMH}+\mathrm{EEBG} 250 \mathrm{mg} / \mathrm{kg}$ & 8 & 5 & 3 & Adenocarcinoma in situ & 62,5 & 37,5 \\
\hline DMH + EEBG 500 mg/kg & 8 & 3 & 5 & Adenoma & 37,5 & 62,5 \\
\hline
\end{tabular}

DMH: valores de dosis efectiva media; EEBG: Baccharis genistelloides.

libres ${ }^{(12)}$, redujeron la mutación genética de microorganismos previniendo la caries dental ${ }^{(13)}$, y que presentaron actividad antitumoral en el modelo de infección de discos de patata con A. tumefaciens, los que transforman las células normales de la patata en células tumorales ${ }^{(14)}$.

En el presente estudio in vivo se ha demostrado que el EEBG, probablemente en virtud a la presencia de flavonoides (tabla 1), ha mostrado tener un efecto protector dosis dependiente en el desarrollo de cáncer de colon en ratas machos inducido con 1,2 dimetilhidrazina (DMH), así como una reducción de los niveles de óxido nítrico (NO) y marcadores de estrés oxidativo.
El estudio fitoquímico del EEBG reveló la presencia de alcaloides en abundante cantidad. Se sabe que los alcaloides son solubles en solventes polares como el etanol, tanto en su forma de sal como de base libre ${ }^{(15)}$.

Si bien es cierto que algunos alcaloides han mostrado tener actividad antioxidante -como en el caso de alcaloides de la vinca y bocconia arbórea ${ }^{(16)}$, , pero no pertenecen a la familia Asteraceae, por lo que no se puede afirmar que son los mismos alcaloides que contiene el Baccharis genistelloides.

Se conoce alcaloides comunes de la familia Asteraceae; tenemos a los alcaloides pirrolizidínicos, los cuales son

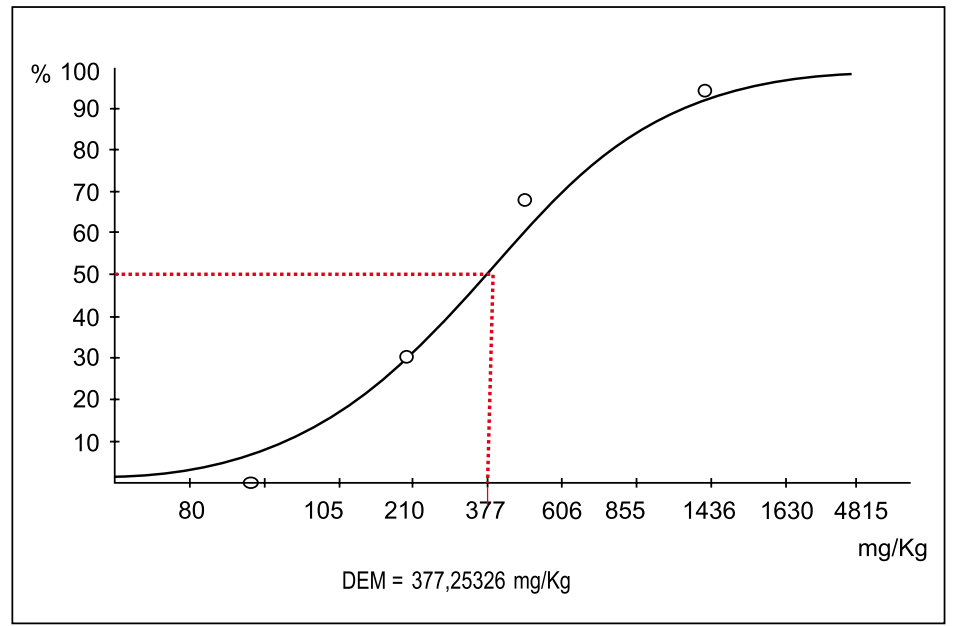

Figura 3. Dosis efectiva media del extracto etanólico de las hojas de Baccharis genistelloides (carqueja) sobre el cáncer de colon inducido en ratas con DMH. Límite de confianza 95\% $(238,71 \mathrm{mg} / \mathrm{kg}-837,90 \mathrm{mg} / \mathrm{kg})$. hepatotóxicos y carecen de actividad farmacológica ${ }^{(17)}$. Hasta el momento, se ha informado sobre la presencia del alcaloide 'bacariana', el cual es común en el Baccharis coridifolia y Baccharis megapotamica, y al cual solo se le atribuye una actividad tóxica.

Tomando en cuenta la información antes comentada sobre los alcaloides, se deduce que estarían en gran cantidad en el EEBG y no se desliga la posibilidad que hayan contribuido en coadyuvar al efecto quimioprotector, pero no se dejaría de lado que la presencia de flavonoides sean los otros agentes contribuyentes en el efecto, pues hasta la fecha se ha informado que las actividades farmacológicas del género baccharis se deben principalmente a los flavonoides y que estas hipótesis son interesantes para generar investigaciones siguientes o futuras. Pero, el objetivo de la presente investigación fue evaluar el efecto quimioprotector del EEBG sobre el sobre el cáncer de colon.

En el estudio se halló la presencia de flavonoides y compuestos fenólicos en regular cantidad. Estos hallazgos concuerdan con otra comunicación (3), al evaluar el extracto etanólico de toda la planta de Baccharis genistelloides (carqueja) procedente de Brasil y Perú (Cajamarca), donde indican haber encontrado la presencia de flavonoides, chalconas, glucósidos del ácido fenilpropanoico, poliacetilenos, un diterpeno, glucósidos de flavona, compuestos fenólicos. Hasta el momento se sabe que las principales acciones farmacológicas del género Baccharis se deben generalmente a la presencia de flavonoides y terpenos; esto conduce al estudio de flavonoides que van a tener actividad antimutagénica ${ }^{(3)}$. Tanto el cirsiliol, eupatrin, cirsimaritin, genkwanina, rutina y quercetina son algunos de los flavonoides presentes en el género Baccharis ${ }^{(12)}$, donde principalmente la quercetina sería la que tendría actividad reductora de iones superóxido (ONOO-) y lipoperoxidación ${ }^{(4,12)}$.

La 1,2-dimetilhidrazina (DMH) es el agente procarcinogenético más usado para producir cáncer de colon experimental en roedores; se metaboliza en el 
Tabla 3. Efecto del extracto etanólico de hojas de Baccharis genistelloides (carqueja) sobre los niveles de nitritos en plasma de ratas. Los valores están dados en promedio +/- DE. Grupos de 8 ratas cada uno, comparados con prueba de diferencia significativa mínima, con una $p<0,05$.

\begin{tabular}{llccc}
\hline Variable & \multicolumn{1}{c}{ Tratamiento } & Valor medio & \multicolumn{2}{c}{ Intervalo de confianza 95\% } \\
\cline { 4 - 5 } & & & inferior & superior \\
\hline & Normal & $3,51+1,39$ & 2,87 & 4,05 \\
& Baccharis genistelloides $100 \mathrm{mg} / \mathrm{kg}$ & $2,99+1,77$ & 2,05 & 3,75 \\
Nitritos & Dimetilhidrazina (DMH) $20 \mathrm{mg} / \mathrm{kg}$ & $5,89+1,48$ & 4,92 & 6,15 \\
micromol/L & B. genistelloides $100 \mathrm{mg} / \mathrm{kg}+\mathrm{DMH}$ & $2,89+1,28$ & 2,03 & 3,35 \\
p<0,05 & B. genistelloides $250 \mathrm{mg} / \mathrm{kg}+\mathrm{DMH}$ & $3,18+1,22$ & 2,52 & 4,12 \\
& B. genistelloides 500 mg/kg + DMH & $2,58+0,76$ & 1,91 & 3,05 \\
\hline
\end{tabular}

hígado, dando como resultado al intermediario azoximetano, el cual finalmente se metaboliza a un agente carcinogenético 'ión diazonium' ${ }^{(18)}$, que, por alquilación de macromoléculas como el ADN, produce mutación ${ }^{(18)}$ y también es capaz de producir efectos tóxicos en lugares diferentes del tumor ${ }^{(19)}$. También, se ha demostrado que la $\mathrm{DMH}$ potencia la acción de dos enzimas, favoreciendo la formación de cáncer de colon: la B-glucoronidasa que hidroliza los glucorónidos biliares en el colon produciendo agliconas -compuestos tóxicos cancerígenos- y la musinasa, que hidroliza a la mucina responsable de la protección de la mucosa del colon ${ }^{(9)}$. De esta forma se entiende que el incremento de ambas enzimas frente a la administración de DMH potencia la actividad cancerígena.

Según la Sociedad Nacional del Cáncer (National Cancer Society), en el cáncer de colon se presenta un fenómeno que consiste en una reducción severa del peso en el paciente, denominado 'caquexia relacionada al cáncer'. Esta las células cancerosas utilizan mucho del suministro de energía del cuerpo; además, a que el exceso de citoquinas (común en el cáncer) ejerce alteración sobre el metabolismo, en donde el procesamiento de comida y construcción reducción de peso puede deberse a que

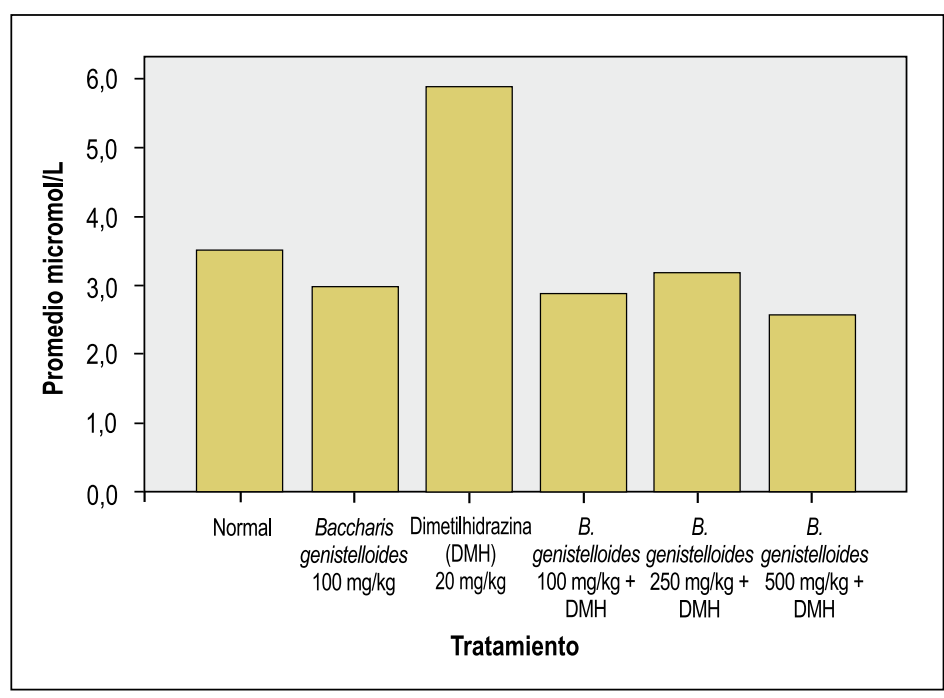

Figura 4. Niveles de nitritos en plasma luego de 22 semanas de administración de extracto etanólico de hojas de Baccharis genistelloides (carqueja) en ratas con cáncer de colon inducido con DMH. de proteínas se ven afectados. Hasta el momento, se sabe que el factor de trascripción AP-1 presenta una regulación diferencial en el músculo esquelético durante el crecimiento tumoral, lo que sugiere que este factor está implicado en el desarrollo de la caquexia asociada al cáncer ${ }^{(20)}$.

$\mathrm{Al}$ inicio del estudio se observó que el peso corporal en todos los grupos no presentó diferencia significativa $(p>0,05)$. A partir de la séptima semana, se observó la reducción de peso severa (caquexia) en los grupos que recibieron $\mathrm{DMH}$, evidenciándose mejor en la semana 14 hasta llegar a la semana 22, donde se observó macroscópicamente que el promedio de peso corporal en los animales estudiados disminuyó en el grupo tratado solo con DMH al compararlo con el grupo normal y los que recibieron EEBG mas DMH, observándose un mayor incremento de peso en el grupo normal y el grupo que recibió solo EEBG (figura 1). Se notó que los animales que solo recibieron DMH presentaron una marcada reducción del peso corporal.

El efecto quimioprotector de las distintas dosis del extracto etanólico de las hojas de Baccharis genistelloides frente a adenomas y adenocarcinomas inducidos por DMH fue mejor a dosis de $500 \mathrm{mg} /$ $\mathrm{kg}$, porque solo hubo incremento de glándulas colónicas e infiltrado celular crónico, con presencia de mononucleares en el parénquima y zonas de hipoplasia glandular con abundantes macrófagos (figura 2f). Con la dosis de $250 \mathrm{mg} / \mathrm{kg}$ se apreció adenocarcinoma in situ, indicando que no hubo compromiso fuera de la membrana basal (figura 2e), mientras que a la dosis de $100 \mathrm{mg} / \mathrm{kg}$ se observó adenocarcinoma glandular (figura 2d). Sin embargo, los animales que solamente recibieron tóxico sin Baccharis genistelloides presentaron desorganización celular, llegando a provocar adenocarcinoma infiltrante mixto, glandular y escamoso (figura 2c), con niveles elevados de oxido nítrico y marcador de estrés oxidativo encontrados en la presente investigación (figuras 4 y 5 ). Por lo anterior, el efecto quimioprotector de la planta fue dosis dependiente y podría deberse a que los flavonoides contienen 
Tabla 4. Efecto del extracto etanólico de hojas de Baccharis genistelloides sobre los niveles de malondialdehido en plasma de ratas. Los valores están dados en promedio + DE. Grupos de 8 ratas cada uno, comparados con prueba de diferencia significativa mínima, con una $p<0,05$.

\begin{tabular}{llccc}
\hline Variable & \multicolumn{1}{c}{ Tratamiento } & Valor medio & \multicolumn{2}{c}{ Intervalo de confianza 95\% } \\
\cline { 4 - 5 } & & & inferior & superior \\
\hline & Normal & $6,83+0,51$ & 6,23 & 7,11 \\
& Baccharis genistelloides $100 \mathrm{mg} / \mathrm{kg}$ & $9,60+1,56$ & 8,56 & 10,12 \\
Malondialdehido & Dimetilhidrazina (DMH) $20 \mathrm{mg} / \mathrm{kg}$ & $6,33+0,80$ & 5,68 & 7,15 \\
micromol/L & B. genistelloides $100 \mathrm{mg} / \mathrm{kg}+\mathrm{DMH}$ & $6,43+1,08$ & 5,51 & 7,39 \\
p<0,05 & B. genistelloides $250 \mathrm{mg} / \mathrm{kg}+\mathrm{DMH}$ & $6,67+0,58$ & 6,12 & 7,01 \\
& B. genistelloides $500 \mathrm{mg} / \mathrm{kg}+\mathrm{DMH}$ & $5,67+0,57$ & 5,16 & 6,11 \\
\hline
\end{tabular}

en su estructura química un número variable de grupos hidroxilo fenólicos y excelentes propiedades de quelación del hierro y otros metales de transición, lo que les confiere una gran capacidad antioxidante $^{(21)}$.

Por lo expuesto, los flavonoides desempeñan un papel esencial en la protección frente a los fenómenos de daño oxidativo y se ha observado que tienen efectos terapéuticos en un elevado número de patologías, incluyendo la cardiopatía isquémica, la aterosclerosis o el cáncer (22). Hasta el momento se ha demostrado en estudio in vivo que, al administrar el flavonoide quercetina por vía oral en dosis altas, inhibe la carcinogénesis del cáncer de colon en ratas machos inducido por azoximetano (AOM) ${ }^{(4)}$.

El extracto etanólico de las hojas de Baccharis genistelloides (carqueja) ha mostrado brindar actividad quimioprotectora del cáncer de colon inducido en ratas machos por DMH a una dosis efectiva media de $377,25 \mathrm{mg} / \mathrm{kg}$.

La cuantificación de óxido nítrico para esta investigación fue mediante el dosaje de nitritos en plasma ${ }^{(10)}$. El óxido nítrico es una molécula que se presenta en exceso en el cáncer de colon

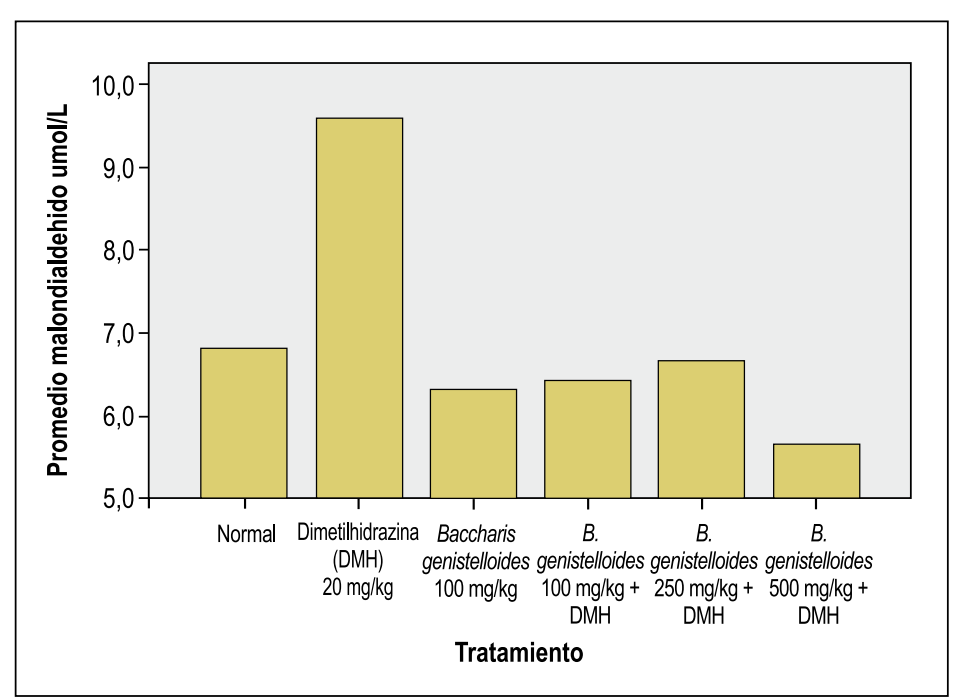

Figura 5. Niveles de malondialdehído en plasma luego de 22 semanas de administración de extracto etanólico de hojas de Baccharis genistelloides (carqueja) en ratas con cáncer de colon inducido con DMH. inducido en roedores y en carcinomas humanos, llegando a ser un indicador de células colónicas tumorales ${ }^{(9)}$. Al parecer, los macrófagos que se infiltran en el tumor podrían generar factor de crecimiento vascular del endotelio (VEGF) y que el $\mathrm{NO}$ es el que regula la actividad del VEGF en la angiogénesis y el reforzamiento de la permeabilidad vascular y retención macromolecular y agentes lipídicos (EPR) propio de los tumores ${ }^{(23)}$. Adicionalmente, el ión superóxido $\left(\mathrm{O}_{2}^{-}\right)$podría generarse de varias maneras en los tejidos tumorales: a causa del reclutamiento de macrófagos y neutrófilos ${ }^{(24)}$, vía xantino oxidasa en tumores sólidos ${ }^{(25)}$ y por acción de la óxido nítrico sintetasa (NOS), en ausencia de L-arginina ${ }^{(26)}$. El $\mathrm{NO}$ y el $\mathrm{O}_{2}{ }^{-}$pueden reaccionar simultáneamente de forma acelerada, generando otro ión mucho más activo y lesivo, peroxinitrito $\left(\mathrm{ONOO}^{-}\right)^{(27)}$, el cual estaría involucrado directamente con la EPR, ya que este sería el responsable de la activación de metaloproteinasas de la matriz (MMPs), que tienen rol importante en la invasión tumoral, metástasis y angiogénesis. La activación de las MMPs generaría una cascada de proteinasas, las que producirían potentes agentes vasoactivos como la bradiquinina ${ }^{(7)}$, favoreciendo la formación de los tumores colónicos.

Por otro lado, se observó que la óxido nítrico sintetasa inducible (iNOS) se encuentra incrementada en la mutación del gen $\mathrm{K}$-ras, produciendo la formación de criptas fósiles aberrantes (CFA) ${ }^{(5)}$ y que, además, la inhibición del iNOS disminuye el crecimiento y proliferación de los tumores en el colon ${ }^{(28)}$.

El NO también refuerza la producción de la COX-2 en el cáncer de colon, el cual por medio de la $\mathrm{GE}_{2}$ producirá angiogénesis ${ }^{(29)}$. En la carcinogénesis del colon inducida con AOM, el COX-2 se encuentra incrementado ${ }^{(28)}$. Se ha observado que los inhibidores de iONS no solo reducen el iONS sino también la activación de la COX-2 ${ }^{(30)}$. Ha quedado establecido que los inhibidores de iONS pueden suprimir la formación de CFA ${ }^{(31)}$. Por lo tanto, la reducción del NO también reducirá la producción de COX-2 y por ende la angiogénesis. 
Como era de esperarse en este estudio, los animales que recibieron solamente DMH fueron los que presentaron mayor nivel de $\mathrm{NO}$ en plasma y los que recibieron tratamiento con $500 \mathrm{mg} / \mathrm{kg}$ de EEBG presentó el menor incremento de $\mathrm{NO}$ con respecto a los animales que recibieron dosis menores de EEBG (tabla 3 y figura 4) y una mejor respuesta histopatológica en cuanto a la formación de adenocarcinomas. Estos estudios evidencian la trascendencia del $\mathrm{NO}$ en los adenocarcinomas en células colónicas y que la reducción de este es importante para prevenir la formación, crecimiento y proliferación de tumores.

El estrés oxidativo puede ser cuantificado mediante el indicador malondialdehido $\mathrm{nmol} / \mathrm{mL}$ (MDA), que es una sustancia producto de la reacción con el ácido tiobarbitúrico.

El nivel de MDA cuantificado en las ratas en tratamiento fue superior en las que recibieron solamente $\mathrm{DMH}$, mientras que en las que recibieron tratamientos con EEBG descendieron significativamente, siendo más marcado en el grupo que recibió dosis de $500 \mathrm{mg} / \mathrm{kg}$ (tabla 5, figura 10). Estos resultados indican una reducción del estrés oxidativo por el tratamiento con EEBG, lo cual es favorable para la protección del colon, ya que frente a un estrés oxidativo severo se puede desencadenar una carcinogénesis por especies reactivas como el ión superóxido $\left(\mathrm{O}_{2}^{-}\right)$y el ión hidroxilo $\left(\mathrm{OH}^{-}\right)$, que son liberados a la circulación sanguínea, resultando en el plasma una lipoperoxidación en ratas tratadas con DMH. Desde que se sabe que el estrés oxidativo eleva la producción de prostaglandina, y además interrumpe la producción de la glutatión peroxidasa conllevando al cáncer de colon, se ha tomado importancia en productos que modularían el estrés oxidativo ${ }^{(30)}$.

En este estudio se observó que la lipoperoxidación asociada con el incremento de los niveles de óxido nítrico en la circulación sanguínea refuerza la inducción de cáncer con DMH en ratas y que EEBG estaría contribuyendo en la prevención de adenocarcinomas colónicos. Los mecanismos implicados en dicha prevención serian: a) reduciendo la síntesis de óxido nítrico y lipoperoxidación a nivel de las células colónicas; b) previniendo la formación de peroxinitritos, mutación del gen K-ras y síntesis de COX-2 responsable de la angiogénesis; $y, c)$ reduciendo la acción de las enzimas B-glucoronidasa y mucinasa.

Al haberse demostrado que extracto etanólico de Baccharis genistelloides (carqueja) tiene efecto quimioprotector en el colon de ratas, se recomienda ampliar estudios de genotoxicidad, toxicidad y seguridad a dosis repetidas con tiempos prolongados, a fin de evidenciar un producto natural con potencial quimioprotector del cáncer de colon, ya que se estaría contribuyendo en mostrar una alternativa terapéutica a base de productos naturales tales como Bacharis genistelloides (carqueja), a fin de coadyuvar con el mejoramiento de los signos y síntomas del cáncer de colon.

\section{REFERENCIAS BIBLIOGRÁFICAS}

1. Jemal A, Murray T, Ward E, Samuels A, Tiwari R, Ghafoor A. Cancer statistics. CA Cancer J Clin. 2005;55:10-30.

2. Arroyo J, Ronceros S, Bonilla P, Quino M, Ráez E, Li E y col. Efecto de Baccharis genistelloides (Carqueja) sobre neoplasia gástrica inducida en ratas. An Fac med. 2002;63 Supl 1:26.

3. Gonzaga V, Costa I, Pizzolatti M. Género Baccharis (Asteraceae). Aspectos químicos e biológicos. Química Nova. 2005;28(1):512-6.

4. Dihal A, Boer V, Van der Woude H, Tilburgs C. Quercetin, but not its glycosidated conjugate rutin, inhibits azoxymethane-induced colorectal carcinogénesis in F344 rats. J Nutr. 2006;136(11):2862-7.

5. Bissonnette $M$, Khare $S$, Von Lintig F, Wali R, Lan Nguyen, Zhang $Y$, et al. Mutational and nonmutational activation of $\mathrm{p} 21$ ras in rat colonic azoxymethane induced tumors: effects on mitogenactivated protein kinase, cyclooxygenase-2, and cyclin D11. J Cancer Res. 2000;60:4602-9.

6. Takahashi M, Mutoh M, Shoji Y, Kamanaka Y, Naka M, Maruyama T, et al. Transfection of K-rasAsp12 cDNA markedly elevates IL-1b- and lipopolysaccharide-mediated inducible nitric oxide synthase expression in rat intestinal epithelial cells. Oncogene. 2003;22:7667-76.

7. Wu J, Akaike T, Hayashida K, Okamoto T, Okuyama A, Maeda $\mathrm{H}$. Enhanced vascular permeability in solid tumor involving peroxynitrite and matrix metalloproteinases. Jpn J Cancer Res. 2001;92:439-51.
8. Lock de Ugaz 0. Investigación Fitoquímica. Métodos en el Estudio de Productos Naturales. 2da edición. Lima: Fondo Editorial Pontificia Universidad Católica del Perú; 1994. p. 33-5.

9. Namasivayam N, Vaiyapuri M, Venugopal M. Effect of coconut cake on the bacterial enzyme activity in 1,2 - dimethyl hydrazine induced colon cancer. Clin Chim Acta. 2004;342:20310.

10. Katrina M, Miranda, Michael G, David A. A rapid, simple spectrophotometric method for simultaneous detection of nitrate and nitrite. Nitric Oxide. 2001;5(1):62-71.

11. Buege J, Aust S. Microsomal lipid peroxidation. Methods in Enzymology. 1978;52:302-10.

12. Tapia A, Rodriguez J, Theoduloz C, López S, Egly G, Schmeda G. Free radical scavengers and antioxidants from Baccharis grisebachii. J Ethnopharmacol. 2004;95:155-61.

13. Leitao D, Filho A, Polizello A, Bastos J, Spadaro A. Comparative evaluation of in-vitro effects of Brazilian green propolis and Baccharis dracunculifolia extracts on cariogenic factors of Streptococcus mutans. Biol Pharm Bull. 2004;27(11):1834-9.

14. Mongelli E, Desmarchelier C, Rodriguez J, Coussio J, Ciccia G. In vitro antioxidant and cytotoxic activity of extracts of Baccharis coridifolia DC. J Ethnopharmacol. 1997;58(3):157-63.

15. Soto M. Introducción a los alcaloides [Internet]. San Francisco: Scribd [citado el 20 de julio de 2008]. Disponible en: http://www.scribd.com/ doc/499723/ALCALOIDES-por-QF-Marilu-RoxanaSoto-Vasquez

16. Mendoza I, Yoldi T, Martínez G. Actividad antioxidante del clorhidrato de triacetonamina in vitro. Rev Cubana Invest Biomed. 2003;22(2):81-9.

17. Carreteno A. Alcaloides: Aspectos generales (I). Panorama actual Med. 2001;25(41):222-7.

18. Sohn 0, Fiala E, Requeijo S, Weisburger J, Gonzalez F. Differential effects of CYP2E1 status on the metabolic activation of the colon carcinogens azoxymethane and methylazoxymethanol. Cancer Res. 2001;61:8435-40.

19. Bobek P, Galbavy S, Mariassyova M. The effect of red beet (Beta vulgaris var. rubra) fiber on alimentary hypercholesterolemia and chemically induced colon carcinogenesis in rats. Nahrung. 2000;44:184-7.

20. Argilés J, Busquets S, López-Soriano F, Figueras M. Fisiopatología de la caquexia neoplásica. Nutrición Hospitalaria. 2006;21:4-9.

21. Havsteen B. Flavonoids. A class of natural products of high pharmacological potency. Biochem Pharmacol. 1983;32:1141-8.

22. Jang M, Cai L, Udeani G. Cancer chemopreventive activity of resveratrol. Science. 1997;275:21822.

23.Xiong M, Elson G, Legarda D, Leibovich SJ. Production of vascular endothelial growth factor 
by murine macrophages: regulation by hypoxia, lactate, and the inducible nitric oxide synthase pathway. Am J Pathol. 1998;153:587-98.

24. Miller R, Britigan B. The formation and biologic significance of phagocyte-derived oxidants. J Invest Med. 1995;43:39-49.

25. Akaike T, Suga M, Maeda H. Free radicals in viral pathogenesis: molecular mechanisms involving superoxide and NO. Proc Soc Exp Biol Med. 1998;217:64-73.

26. Xia Y, Dawson V, Dawson T, Snyder S, Zweier J. Nitric oxide synthase generates superoxide and nitric oxide in arginine-depleted cells leading to peroxynitrite mediated cellular injury. Proc Natl Acad Sci. 1996;93:6770-4.

27. Beckman J, Koppenol W. Nitric oxide, superoxide, and peroxynitrite: the good, the bad, and the ugly. Am J Physiol. 1996;271:1424-37.
28. Takahashi M, Mutoh M, Shoji Y, Sato H, Kamanaka $\mathrm{Y}$, Mayurama T, et al. Suppressive effect of an inducible nitric oxide inhibitor, ONO - 1714 on AOM - induced rat colon carcinogenesis. Nitric Oxide. 2006;14:130-6.

29. Cianchi F, Cortesini C, Fantappie 0, Messerini L, Sardi L, Lasagna N, et al. Cyclooxygenase-2 activation mediates the proangiogenic effect of nitric oxide in colorectal cancer. Clin Cancer Res. 2004;10:2694-704

30. Rao CV, Indranie C, Simi B, Manning PT, Connor JR, Reddy BS. Chemopreventive properties of a selective inducible nictric oxide synthase inhibitor in colon carcinogenesis, administered alone or in combination with celecoxib, a selective cyclooxygenase-2 2 inhibitor. Cancer Res. 2002;62:165-70.

31. Kawamori T, Takahashi M, Watanabe K, Ohta T, Nakatsugi S, Sugimura T, et al. Suppression of azoxymethane-induced colonic aberrant crypt foci by a nitric oxide synthase inhibitor. Cancer Lett. 2000;148:33-7.

Manuscrito recibido el 16 de mayo de 2010 y aceptado para publicación el 10 de junio de 2010.

Correspondencia:

Dr. Jorge Arroyo Acevedo

Sección Farmacología

Facultad de Medicina, UNMSM.

Av. Grau 755.

Lima 1, Perú.

Correo-e: jorgeluis_arroyoacevedo@yahoo.es 